\title{
Searching for Black Hole Candidates by LAMOST and ASAS-SN
}

 \\ $\mathrm{Yu} \mathrm{Bai}^{2}$ (D), Yi-Ze Dong ${ }^{1,4}$, Hao-Tong Zhang ${ }^{2}$, Ya-Juan Lei ${ }^{2}$, Junfeng Wang ${ }^{1}$ (D), Jianfeng Wu ${ }^{1}$, and Jifeng Liu ${ }^{2,5}$ \\ ${ }^{1}$ Department of Astronomy, Xiamen University, Xiamen, Fujian 361005, People’s Republic of China; guwm@xmu.edu.cn \\ ${ }^{2}$ National Astronomical Observatories, Chinese Academy of Sciences, Beijing 100012, People's Republic of China \\ ${ }^{3}$ Infrared Processing and Analysis Center, California Institute of Technology, Pasadena, CA 91125, USA \\ ${ }^{4}$ Department of Physics, University of California, Davis, CA 95616, USA \\ ${ }^{5}$ College of Astronomy and Space Sciences, University of Chinese Academy of Sciences, Beijing 100049, People's Republic of China \\ Received 2019 June 27; revised 2019 August 27; accepted 2019 September 12; published 2019 October 10
}

\begin{abstract}
Most dynamically confirmed stellar-mass black holes (BHs) and their candidates were originally selected from X-ray outbursts. In the present work, we search for BH candidates in the Large Sky Area Multi-Object Fiber Spectroscopic Telescope (LAMOST) survey using the spectra along with photometry from the All Sky Automated Survey for SuperNovae (ASAS-SN), where the orbital period of the binary may be revealed by the periodic light curve, such as the ellipsoidal modulation type. Our sample consists of nine binaries, where each source contains a giant star with large radial velocity variation $\left(\Delta V_{\mathrm{R}} \gtrsim 70 \mathrm{~km} \mathrm{~s}^{-1}\right)$ and periods known from light curves. We focus on the nine sources with long periods ( $T_{\mathrm{ph}}>5$ days) and evaluate the mass $M_{2}$ of the optically invisible companion. Since the observed $\Delta V_{\mathrm{R}}$ from only a few repeating spectroscopic observations is a lower limit of the real amplitude, the real mass $M_{2}$ can be significantly higher than the current evaluation. It is likely an efficient method to place constraints on $M_{2}$ by combining $\Delta V_{\mathrm{R}}$ from LAMOST and $T_{\mathrm{ph}}$ from ASAS-SN, particularly by the ongoing LAMOST Medium Resolution Survey.
\end{abstract}

Unified Astronomy Thesaurus concepts: Stellar mass black holes (1611); Compact binary stars (283); Stellar photometry (1620); Radial velocity (1332); Stellar spectral types (2051)

\section{Introduction}

It is well known that three types of compact objects in the universe are white dwarfs, neutron stars, and black holes (BHs). Since an isolated $\mathrm{BH}$ does not produce electromagnetic radiation, most confirmed stellar-mass BHs and candidates were found in binaries (Remillard \& McClintock 2006). For a binary system composed of a $\mathrm{BH}$ and an optically visible star filling its Roche lobe, the matter from the star can be accreted by the BH through the inner Lagrange point. In such cases, an accretion disk is formed and X-ray emission is produced from the disk. Thus, such a BH binary system can be detected by X-ray telescopes. However, the number of confirmed $\mathrm{BHs}$ and $\mathrm{BH}$ candidates found by this method is less than a hundred (Corral-Santana et al. 2016), which is far below the number of BHs that are thought to exist in our Galaxy (e.g., Brown \& Bethe 1994).

New methods are required to search for more $\mathrm{BH}$ candidates. For binaries with unknown orbital periods, Gu et al. (2019) proposed a method to search for $\mathrm{BH}$ candidates from optical observations. The method is based on the assumption that the radius $R_{1}$ of the optically visible star is no more than the corresponding Roche-lobe radius $R_{\mathrm{L} 1}$. On the other hand, once the orbital period $P_{\text {orb }}$ can be derived (such as being revealed by the periodic light curves), we can obtain the well-known mass function (refer to Equation (5) in Section 3.2) and therefore place better constraints on the optically invisible companion. In a BH binary, if the ratio $R_{1} / R_{\mathrm{L} 1}$ is not far below unity, the companion may be pulled into a waterdrop shape due to the strong gravity of the $\mathrm{BH}$. The deformed star will present a periodic light curve with the ellipsoidal modulation (Morris 1985). Thus, the light curve may reveal the orbital period $P_{\text {orb }}$ of the system and is helpful to the constraints of $M_{2}$.

The Large Sky Area Multi-Object Fiber Spectroscopic Telescope (LAMOST) provides nearly 10 million stellar spectra in the Data Release 6 and about 480 thousand low-resolution stellar spectra in the Data Release 7. Furthermore, it has radial velocity to a precision of better than $5 \mathrm{~km} \mathrm{~s}^{-1}$ (Deng et al. 2012). We can derive many key parameters $\left(T_{\text {eff }}, \log g\right.$, and $\left.[\mathrm{Fe} / \mathrm{H}]\right)$ and heliocentric radial velocity $V_{\mathrm{R}}$ from the spectra (Zong et al. 2018). In addition, the All Sky Automated Survey for SuperNovae (ASAS-SN) monitors the entire visible sky to a depth of $V \lesssim 17 \mathrm{mag}$ for bright supernovae and other transients. For instance, the ASAS-SN $V$-band light curves of around 412 thousand variable stars were studied by Jayasinghe et al. (2019).

The aim of this paper is to introduce the method to search for BH candidates by combining the LAMOST spectra and the ASAS-SN photometry. We will introduce the data selection from LAMOST in Section 2. The analyses and results of our sample are shown in Section 3. Conclusions and discussion are presented in Section 4.

\section{Data Selection}

The present work focuses on binaries with a giant star. For a giant star, the variation of radial velocity in the same night is usually negligible due to its large size, and therefore its orbital period is relatively long. We select a sample of binaries containing a giant star from LAMOST Data Release 6 and LAMOST Data Release 7 with the following criteria:

$$
\left\{\begin{array}{l}
\mathrm{S} / \mathrm{N}_{(\mathrm{g})}>10 \text { (signal-to-noise in the } g \text { band), } \\
3800 \mathrm{~K}<T_{\mathrm{eff}}<5300 \mathrm{~K}, \\
1.5 \mathrm{dex}<\log g<3.5 \mathrm{dex} \\
-1.0 \mathrm{dex}<[\mathrm{Fe} / \mathrm{H}]<0.5 \mathrm{dex}, \\
\text { single-lined spectra only. }
\end{array}\right.
$$

Furthermore, the selected sources have at least two-night exposures in the LAMOST database, and the largest radial 
velocity variation $\Delta V_{\mathrm{R}} \gtrsim 70 \mathrm{~km} \mathrm{~s}^{-1}$. Consequently, we obtain a sample of 43 single-lined binaries. In addition, the sources without Gaia parallax or with negative parallax have been removed (Jayasinghe et al. 2019; Ziaali et al. 2019). We crossmatch the sources with the ASAS-SN Sky Patrol ${ }^{6}$ database (Shappee et al. 2014; Kochanek et al. 2017), and therefore we derive a sample of 17 binaries with periods longer than 5 days (the reason is given in the fourth paragraph of Section 3.1). We refer to the signal-to-noise ratio $(\mathrm{S} / \mathrm{N})$ in Equation (6) from Hartman \& Bakos (2016) to measure the significance for peaks identified in the periodogram. Finally, we obtain nine sources with $\mathrm{S} / \mathrm{N}\left(T_{\mathrm{ph}}\right)>30$ as our sample, which are shown in Table 1. We also cross-match our sample with simbad in $5^{\prime \prime}$, and find that only source number 3 has X-ray information (refer to Section 3.1).

Since the sources in our sample are all with single-lined spectra, the unseen object in a binary is therefore either a compact object or a much fainter star, roughly speaking, less than $10 \%$ of the luminosity of the observed giant star. The luminosity is shown in column 14 of Table 1 , which is calculated by the apparent magnitude from UCAC4 and the parallax from Gaia DR2, where the bolometric correction and extinction have been taken into consideration. It is seen from Table 1 that the luminosity of these sources is less than 100 solar luminosities. If the unseen object is a main-sequence star or a subgiant star with 3 solar masses, it will be more than 30 solar luminosities. Thus, it ought to be observed and the corresponding spectra of the binary should not be the singlelined type. Thus, once $M_{2}>3 M_{\odot}$ is matched, the unseen object has a high possibility of being a $\mathrm{BH}$. In this work, we manage to search for $\mathrm{BH}$ candidates following this spirit. We would point out another possibility that the system is a triple system. For example, if the system consists of a giant star and a pair of 1.5 solar mass stars in a close binary, then the total luminosity of the pair of stars is around 10 solar luminosities, and therefore may be optically invisible.

\section{Results}

\subsection{Period Analyses}

The Lomb-Scargle method (Lomb 1976; Scargle 1981) is a useful statistical tool to extract periodic signals in unevenly spaced data. This method can reflect the intensity of the captured periodic signal on the power peaks. The LombScargle periodogram is calculated and the period corresponding to the highest power was extracted. Then light curves can be folded with the retrieved periods. We searched for the periods for the sources in our sample, and five of them (source numbers 1-3, 7, and 8) have been investigated by $\mathrm{Gu}$ et al. (2019) without any information of the period. The folded light curves for the nine sources are shown in Figure 1. As mentioned in Section 2, only source number 3 was known as a faint X-ray source according to the ROSAT observations (Voges et al. 2000). The light curve of this source in Figure 1 shows that it may have two possibilities. One is an eclipsing binary caused by an accretion disk, which coincides with the X-ray observations. The other possibility is an eclipsing binary of the Algol type where the X-ray emission is related to an active star. From the shape of light curves, the other eight sources in our sample may be either the ellipsoidal modulation type or the

\footnotetext{
6 https://asas-sn.osu.edu
}

eclipsing binary type. We would stress that, for both of these two mechanisms, the periodic variability can reveal the orbital period. Thus, we can evaluate the mass of the unseen object using the orbital period.

We compare our derived period based on the Lomb-Scargle algorithm with that given by ASAS-SN. We found that our results are identical to that from the ASAS-SN website, except for source number 2. The photometric period (55.1046 days) from the ASAS-SN website for this source is exactly twice of ours (27.5509 days). In our opinion, the different periods may result from different folding algorithms. For this source, we adopt our period in the following analyses.

Even though the shape of folded light curves may indicate that the photometric period $T_{\mathrm{ph}}$ is identical with the orbital period $P_{\text {orb}}$, some analyses are required to confirm that. In a binary, the relation between the separation $a$ and $P_{\text {orb }}$ takes the form of

$$
\frac{G\left(M_{1}+M_{2}\right)}{a^{3}}=\frac{4 \pi^{2}}{P_{\mathrm{orb}}^{2}} .
$$

In addition, the Roche-lobe radius of the optically visible star $R_{\mathrm{L} 1}$ can be expressed as (Paczyński 1971)

$$
\frac{R_{\mathrm{L} 1}}{a}=0.462\left(\frac{M_{1}}{M_{1}+M_{2}}\right)^{1 / 3} \text {. }
$$

Based on the reasonable assumption that the radius of the giant star is no larger than the Roche-lobe radius, i.e., $R_{1} \leqslant R_{\mathrm{L} 1}(\mathrm{Gu}$ et al. 2019), and by combining Equations (1) and (2), the following inequality can be derived:

$$
P_{\text {orb }} \geqslant 2 \pi\left[\frac{\left(R_{1} / 0.462\right)^{3}}{G M_{1}}\right]^{1 / 2} .
$$

Thus, there exists a lower limit for the orbital period once $R_{1}$ and $M_{1}$ (or simply the mass density $\rho_{1}$ ) is derived:

$$
P_{\mathrm{orb}}^{\min }=0.369\left(\rho_{1} / \rho_{\odot}\right)^{-1 / 2} \text { days, }
$$

where $\rho_{\odot}$ is the solar density.

As indicated by Equation (4), if the optically visible star is of main sequence, the orbital period can be less than one day, which agrees well with most confirmed BHs in low-mass X-ray binaries. In this work, however, we focus on the cases in which the companion is a late-type giant star, such as a red giant. For instance, given $M_{1}=M_{\odot}$ and $R_{1}=10 R_{\odot}$, Equation (4) results in $P_{\text {orb }}^{\min } \approx 11.7$ days. That is why we have focused on the nine sources with $T_{\mathrm{ph}}>5$ days. Otherwise, the photometric period $T_{\mathrm{ph}}$ is unlikely the orbital period $P_{\text {orb }}$.

For the sources in our sample, apparent periodic variability $(0.1 \sim 0.5 \mathrm{mag})$ has been observed. If the periodic variability is related to the ellipsoidal modulation, then the radius of an optically visible star cannot be far below the corresponding Roche-lobe radius. Consequently, the period $T_{\mathrm{ph}}$ should not be far beyond $P_{\mathrm{orb}}^{\mathrm{min}}$. Figure 2 shows the consistency of the photometric period and the orbital period. A comparison of the observations with our analyses is shown in Figure 2, where the analytic $P_{\mathrm{orb}}^{\min }$ for $R_{1}=R_{\mathrm{L} 1}$ (solid line) is calculated by Equation (4). In addition, since the giant star may not fill its Roche lobe, we also plot the analytic $P_{\text {orb }}$ for $R_{1}=0.5 R_{\mathrm{L} 1}$ (dashed line). The observations for the nine sources are denoted by different symbols, where $M_{1}$ and $R_{1}$ are derived by the stellar evolution model, as mentioned in Table 1 . It is seen from 
Table $\mathbf{1}$
Parameters for the Sources in Our Sample

\begin{tabular}{|c|c|c|c|c|c|c|c|c|c|c|c|c|c|c|c|c|}
\hline No. & $\begin{array}{l}\text { R.A. } \\
\text { (2) }\end{array}$ & $\begin{array}{l}\text { Decl. } \\
\text { (3) }\end{array}$ & $\begin{array}{c}T_{\mathrm{ph}} \\
\text { (days) } \\
(4)\end{array}$ & $\begin{array}{c}\mathrm{S} / \mathrm{N}\left(T_{\mathrm{ph}}\right) \\
(5)\end{array}$ & $\begin{array}{l}T_{\text {eff }, L} \\
(\mathrm{~K}) \\
(6)\end{array}$ & $\begin{array}{c}\log g \\
(\operatorname{dex}) \\
(7)\end{array}$ & $\begin{array}{c}{[\mathrm{Fe} / \mathrm{H}]} \\
(\mathrm{dex}) \\
(8)\end{array}$ & $\begin{array}{l}N_{\text {obs }} \\
(9)\end{array}$ & $\begin{array}{c}\Delta V_{\mathrm{R}} \\
\left(\mathrm{km} \mathrm{s}^{-1}\right) \\
(10)\end{array}$ & $\begin{array}{l}\varpi \\
\text { (mas) } \\
(11)\end{array}$ & $\begin{array}{c}\text { Vmag } \\
(\mathrm{mag}) \\
(12)\end{array}$ & $\begin{array}{c}\text { Kmag } \\
(\mathrm{mag}) \\
(13)\end{array}$ & $\begin{array}{c}L \\
\left(L_{\odot}\right) \\
(14)\end{array}$ & $\begin{array}{c}R_{1} \\
\left(R_{\odot}\right) \\
(15)\end{array}$ & $\begin{array}{c}M_{1} \\
\left(M_{\odot}\right) \\
(16)\end{array}$ & $\begin{array}{c}M_{2} \\
\left(M_{\odot}\right) \\
(17)\end{array}$ \\
\hline 1 & 0.839201575 & 38.51855052 & 11.8608 & 118.56 & $4696 \pm 57$ & $2.65 \pm 0.09$ & $-0.25 \pm 0.05$ & 3 & $93.5 \pm 5.6$ & $0.505 \pm 0.043$ & 12.736 & 9.952 & 50.176 & $8.1_{-0.8}^{+1.5}$ & $1.1_{-0.1}^{+0.3}$ & $0.9_{-0.1}^{+0.2}$ \\
\hline $2^{\mathrm{a}}$ & 3.887105349 & 38.68886824 & 27.5509 & 116.33 & $4301 \pm 44$ & $1.95 \pm 0.07$ & $-0.47 \pm 0.04$ & 4 & $83.7 \pm 6.2$ & $0.379 \pm 0.032$ & 12.665 & 9.641 & 91.627 & $17.8^{+0.6}$ & $0.9^{+1.5}$ & $1.1^{+0.9}$ \\
\hline 3 & 74.05325351 & 54.00589535 & 5.2085 & 55.65 & $4769 \pm 106$ & $2.68 \pm 0.17$ & $-0.31 \pm 0.10$ & 6 & $127.2 \pm 7.8$ & $1.068 \pm 0.034$ & 12.784 & 9.004 & 31.245 & $7.8_{-0.7}^{+3.5}$ & $1.1_{-0.2}^{+0.7}$ & $1.0_{-0.2}^{+0.4}$ \\
\hline $4^{\mathrm{a}}$ & 82.31076394 & 42.09587217 & 9.6409 & 39.49 & $4700 \pm 98$ & $2.97 \pm 0.15$ & $-0.36 \pm 0.09$ & 4 & $70.5 \pm 6.7$ & $1.398 \pm 0.048$ & 12.727 & 9.941 & 6.774 & $5.1^{+2.8}$ & $0.9^{+0.4}$ & $0.5^{+0.2}$ \\
\hline 5 & 93.81977494 & 22.11031808 & 15.5915 & 32.38 & $4796 \pm 25$ & $2.43 \pm 0.04$ & $-0.47 \pm 0.02$ & 2 & $78.1 \pm 5.6$ & $1.090 \pm 0.072$ & 11.362 & 8.051 & 77.445 & $12.0_{-3.4}^{+3.3}$ & $1.4_{-0.5}^{+0.6}$ & $0.9_{-0.2}^{+0.3}$ \\
\hline 6 & 102.0930387 & 21.82487008 & 7.1848 & 37.92 & $5093 \pm 29$ & $3.11 \pm 0.05$ & $-0.27 \pm 0.03$ & 2 & $78.1 \pm 6.6$ & $0.539 \pm 0.042$ & 12.920 & 10.231 & 42.086 & $6.0_{-0.8}^{+0.8}$ & $1.7_{-0.3}^{+0.2}$ & $0.7_{-0.1}^{+0.2}$ \\
\hline 7 & 111.3363737 & 28.06745981 & 28.0117 & 42.72 & $4833 \pm 188$ & $2.75 \pm 0.30$ & $-0.23 \pm 0.18$ & 6 & $85.1 \pm 7.6$ & $0.152 \pm 0.032$ & 14.698 & 12.075 & 82.412 & $8.7_{-1.9}^{+2.8}$ & $1.5_{-0.4}^{+0.6}$ & $1.4_{-0.3}^{+0.5}$ \\
\hline $8^{\mathrm{a}}$ & 169.1246518 & 55.72840217 & 46.8920 & 74.31 & $4191 \pm 102$ & $1.82 \pm 0.16$ & $-0.75 \pm 0.10$ & 3 & $97.8 \pm 5.8$ & $1.086 \pm 0.031$ & 10.638 & 7.377 & 82.415 & $20.8^{+8.1}$ & $0.9^{+0.5}$ & $1.9^{+0.6}$ \\
\hline 9 & 325.3386324 & 28.4225968 & 17.4856 & 40.81 & $4770 \pm 78$ & $2.51 \pm 0.12$ & $-0.15 \pm 0.07$ & 2 & $73.2 \pm 5.2$ & $1.063 \pm 0.036$ & 10.675 & 8.036 & 67.864 & $10.8_{-0.2}^{+4.1}$ & $1.9_{-0.7}^{+0.6}$ & $1.1_{-0.4}^{+0.3}$ \\
\hline
\end{tabular}

Note.

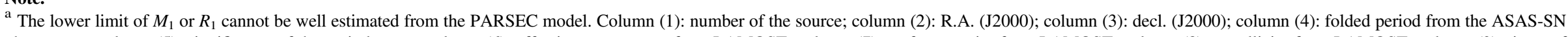

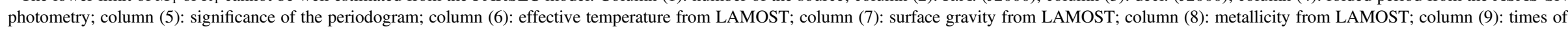



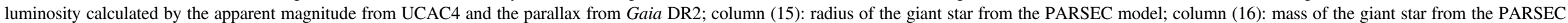
model; and column (17): mass of the invisible star for " $i=60^{\circ}, K_{1}=\Delta V_{\mathrm{R}} / 2$, and $P_{\mathrm{orb}}=T_{\mathrm{ph}}$." 

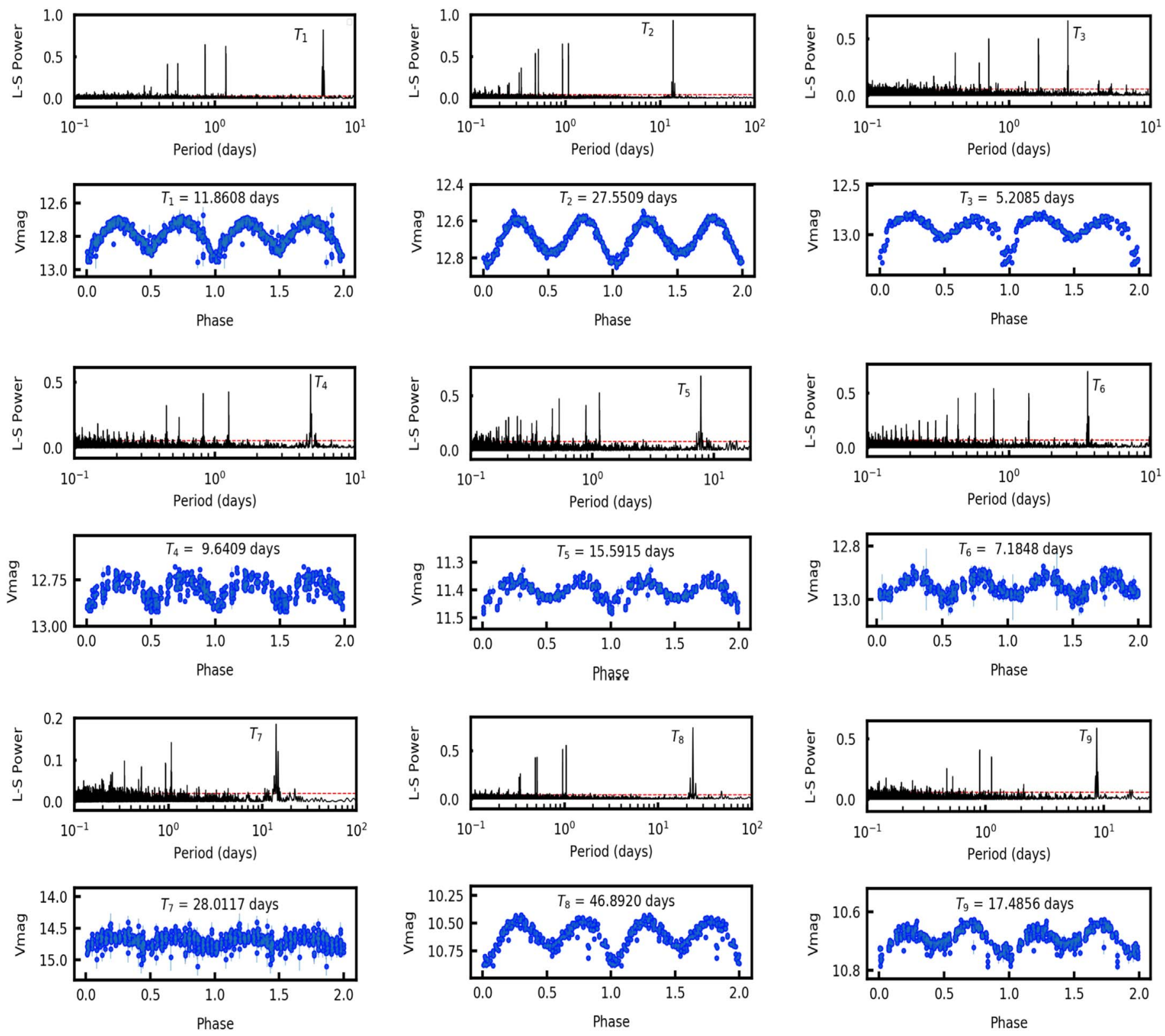

Figure 1. Light curves of the nine sources in Table 1 folded by the Lomb-Scargle algorithm, where the period of variability is shown in each panel.

Figure 2 that all nine sources are well located around the solid line or between the solid line and the dashed line, which indicates that the relation $P_{\mathrm{orb}}=T_{\mathrm{ph}}$ is quite reasonable. As a consequence, we can place better constraints on the mass $M_{2}$ with the values of $P_{\text {orb }}$.

\subsection{Mass Measurement}

We evaluate the mass $M_{1}$ of the nine sources in our sample by the PARSEC model ${ }^{7}$ (Marigo et al. 2008; Bressan et al. 2012), and measure the mass of the optically invisible companion by the equation of mass function. The well-known mass function for $M_{2}$ takes the form (Remillard \& McClintock 2006)

$$
f\left(M_{2}\right) \equiv \frac{M_{2} \sin ^{3} i}{(1+q)^{2}}=\frac{K_{1}^{3} P_{\text {orb }}}{2 \pi G},
$$

\footnotetext{
http://stev.oapd.inaf.it/cgi-bin/cmd_3.1
}

where $K_{1} \geqslant \Delta V_{\mathrm{R}} / 2$ is the semi-amplitude of the giant star and the mass ratio is defined as $q \equiv M_{1} / M_{2}$. In the above equation, once $K_{1}$ and $P_{\text {orb }}$ are given, the mass function $f\left(M_{2}\right)$ can be obtained, and it is certain that $M_{2}>f\left(M_{2}\right)$. In addition, if $M_{1}$ can be derived from the spectra and $\sin i$ is provided, then $M_{2}$ can be well constrained. Referring to the inclination angle of most BH binaries in Corral-Santana et al. (2016), we assume a typical inclination angle of $i=60^{\circ}$ in this work.

As shown in Table $1, M_{1}$ of most sources are in the range of $1 M_{\odot}<M_{1}<2 M_{\odot}$. If $M_{2}>3 M_{\odot}$ can be matched, the source can be regarded as a $\mathrm{BH}$ candidate. However, it is not easy to directly satisfy such a condition. On the other hand, if $M_{2}>M_{1}$ can be matched, according to the rule of stellar evolution, the optically invisible star is likely to be a compact object (except for the Algol case, see below). Thus, we plot four theoretical lines in Figure 3 (from bottom to top) corresponding to $\left(M_{1}=1 M_{\odot}, \quad M_{2}=M_{1}\right), \quad\left(M_{1}=2 M_{\odot}\right.$, 


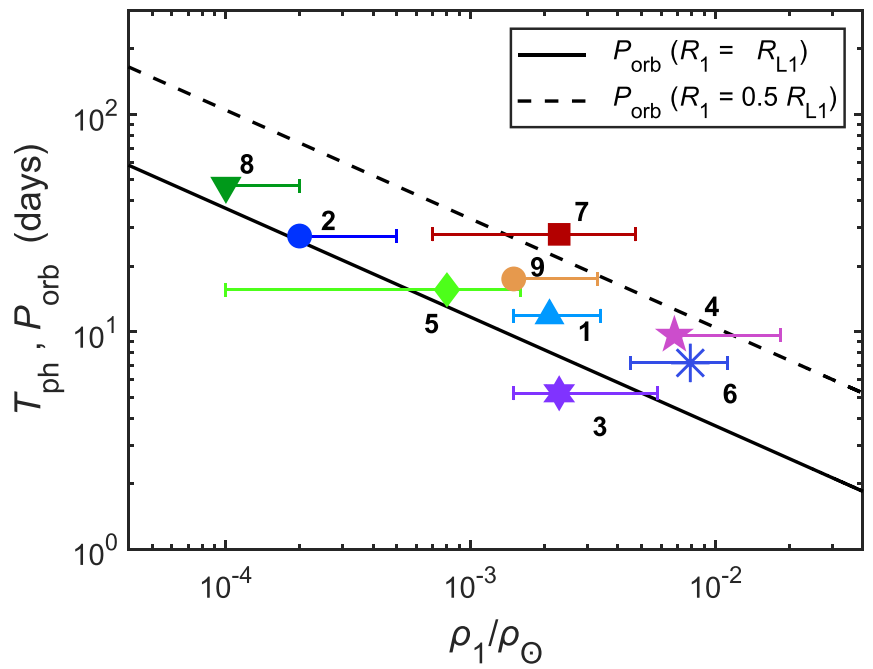

Figure 2. Comparison of the analytic orbital period $P_{\text {orb }}$ (lines) with the observational variability period $T_{\mathrm{ph}}$ (symbols). The solid line represents the lower limit $P_{\mathrm{orb}}^{\mathrm{min}}$ calculated by Equation (4), where the giant star fills its Roche lobe $\left(R_{1}=R_{\mathrm{L} 1}\right)$. The dashed line corresponds to a case in which the Roche lobe is not filled, with $R_{1}=0.5 R_{\mathrm{L} 1}$.

$\left.M_{2}=M_{1}\right), \quad\left(M_{1}=2 M_{\odot}, \quad M_{2}=3 M_{\odot}\right), \quad$ and $\quad\left(M_{1}=1 M_{\odot}\right.$, $\left.M_{2}=3 M_{\odot}\right)$, respectively.

It is seen from Figure 3 that, there is no source above or even in the upper shaded region, which means that there is no strong $\mathrm{BH}$ candidate according to the current observations. On the other hand, source number 8 is located well above the green shaded region, and close to the blue shaded region. Even under the extreme case with the inclination angle is $i=90^{\circ}$, we also derive $M_{2}>M_{1}$. Hence source number 8 is probably a compact object. Whether or not it is a $\mathrm{BH}$ requires follow-up spectroscopic observations to obtain the radial velocity curve. However, we would point out that there exist some binaries like Algol, in which the lower mass star is more evolved than its companion. Thus, mass exchange can allow $M_{2}>M_{1}$ with the more massive star being on the main sequence. In other words, the condition $M_{2}>M_{1}$ may imply a compact star but is not a sufficient condition. In addition, most sources in Table 1 have only two or three observations, and the semi-amplitude of radial velocity $K_{1}$ may be significantly larger than the current $\Delta V_{\mathrm{R}} / 2$. Thus, it is quite possible for the mass $M_{2}$ to be significantly higher than the current evaluation. We therefore use the black arrows in Figure 3 to show such an increase possibility.

\section{Conclusions and Discussion}

In this work, we have proposed a method to search for stellar-mass BH candidates by including the LAMOST spectra and the ASAS-SN photometry, where the orbital period $P_{\text {orb }}$ may be revealed by the periodic light curve. We have obtained a sample of nine single-lined spectroscopic binaries containing a giant star with a large radial velocity variation of $\Delta V_{\mathrm{R}} \gtrsim$ $70 \mathrm{~km} \mathrm{~s}^{-1}$, and the photometric period of the sources satisfy $T_{\mathrm{ph}}>5$ days. Moreover, based on the relation $R_{1} \leqslant R_{\mathrm{L} 1}$, we have checked that $T_{\mathrm{ph}}$ and $P_{\mathrm{orb}}$ are likely identical for the sources in our sample. As a consequence, the mass $M_{2}$ can be better constrained. We have shown that source number 8 is likely to be a compact object. It is worth follow-up spectroscopic observations to check whether it is a $\mathrm{BH}$. Moreover, for

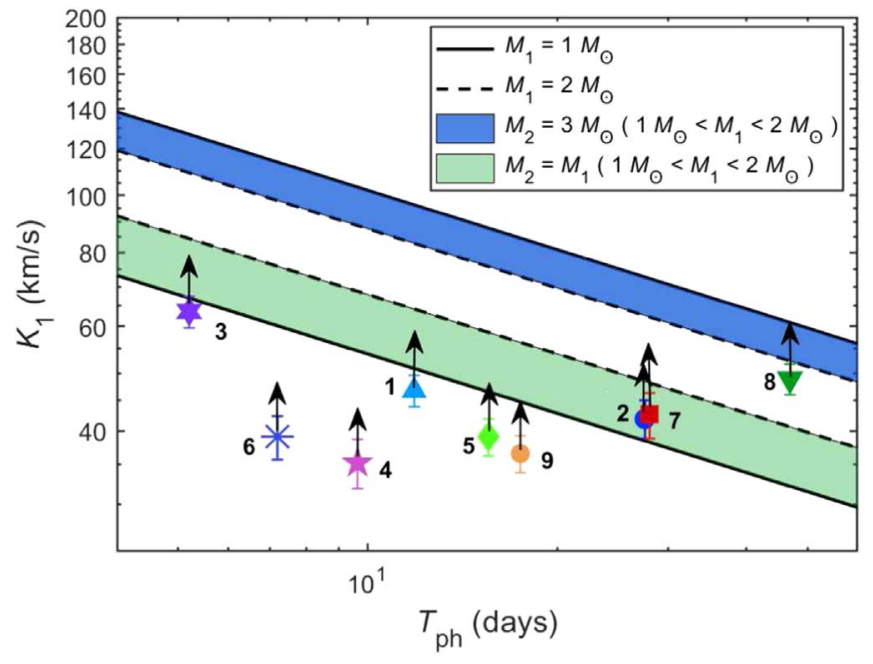

Figure 3. Comparison between analyses and observations in the $K_{1}-T_{\mathrm{ph}}$ diagram, where the semi-amplitude $K_{1}$ is no less than half of the observed maximal variation in a few repeating observations $\left(K_{1} \geqslant \Delta V_{\mathrm{R}} / 2\right)$.

the other sources, the real mass $M_{2}$ can be significantly higher than the current evaluation. Thus, they are also potential $\mathrm{BH}$ candidates. In our opinion, it is an efficient method to constrain $M_{2}$ by combining the LAMOST spectra and the ASAS-SN photometry.

In this work, we have focused on the giant companion. In fact, our method is also valid for the main-sequence star case. Normally, the orbital period $P_{\text {orb }}$ of a main-sequence star is significantly shorter (less than one day, as implied by Equation (4)) for the ellipsoidal modulation type. In such case, the radial velocity variation in the same night can provide crucial information. Thus, once the single exposure spectra of LAMOST are released, many more $\mathrm{BH}$ candidates can be found through our method. On the other hand, the LAMOST Medium Resolution Survey will provide more accurate radial velocity and more repeating exposures (around 60 exposures for a source in the time-domain spectroscopic survey), which enable us to derive a clear radial velocity curve and to make a better constraint on the mass of candidates.

The sources in our sample are binaries with relatively long orbital periods ( $5 \sim 47$ days). However, the Gaia DR2 solution has assumed a single-star model and has mistaken the binary motion itself as part of the parallax, which may result in systematic errors for the parallax and distance. Whether the real parallaxes are larger or smaller than the current values is related to the observational times. For the cases with adequate observations by Gaia, the real parallaxes will be smaller. On the contrary, for the cases with inadequate observations, the results will be quite uncertain. In DR3, the non-single-star model will be considered in the data analysis. In the full release of the nominal mission, the catalog will provide all available variable-star and non-single-star solutions.

We thank Mou-Yuan Sun, Wei-Kai Zong, Xuefei Chen, Zhaoxiang Qi, and Kento Masuda for helpful discussions, and the referee for constructive suggestions that improved the paper. This work was supported by the National Natural Science Foundation of China (NSFC) under grants 11573023, 11603035, 11603038, U1831205, and 11425313, as well as was developed in part at the 2018 Gaia-LAMOST Sprint 
workshop, supported by the National Natural Science Foundation of China (NSFC) under grants 11333003 and 11390372, and the Fundamental Research Funds for the Central Universities under grants 20720190122, 20720190115, and 20720190051. This work has made use of data products from the Guoshoujing Telescope (the Large Sky Area Multi-Object Fiber Spectroscopic Telescope, LAMOST) and the All-Sky Automated Survey for Supernovae (ASAS-SN). LAMOST is a National Major Scientific Project built by the Chinese Academy of Sciences. Funding for the project has been provided by the National Development and Reform Commission. LAMOST is operated and managed by the National Astronomical Observatories, Chinese Academy of Sciences. ASAS-SN is hosted by Las Cumbres Observatory; we thank the Las Cumbres Observatory and its staff for its continuing support of the ASAS-SN project.

\section{ORCID iDs}

Wei-Min Gu (iD https://orcid.org/0000-0003-3137-1851

Hui-Jun Mu (iD https://orcid.org/0000-0001-6589-2220

Song Wang (i) https://orcid.org/0000-0003-3116-5038
Yu Bai (i) https://orcid.org/0000-0002-4740-3857

Junfeng Wang (iD https://orcid.org/0000-0003-4874-0369

\section{References}

Bressan, A., Marigo, P., Girardi, L., et al. 2012, MNRAS, 427, 127 Brown, G. E., \& Bethe, H. A. 1994, ApJ, 423, 659

Corral-Santana, J. M., Casares, J., Muñoz-Darias, T., et al. 2016, A\&A, 587, A61

Deng, L.-C., Newberg, H. J., Liu, C., et al. 2012, RAA, 12, 735

Gu, W.-M., Mu, H.-J., Fu, J.-B., et al. 2019, ApJL, 872, L20

Hartman, J. D., \& Bakos, G. Á 2016, A\&C, 17, 1

Jayasinghe, T., Stanek, K. Z., Kochanek, C. S., et al. 2019, MNRAS, 486, 1907

Kochanek, C. S., Shappee, B. J., Stanek, K. Z., et al. 2017, PASP, 129, 104502

Lomb, N. R. 1976, Ap\&SS, 39, 447

Marigo, P., Girardi, L., Bressan, A., et al. 2008, A\&A, 482, 883

Morris, S. L. 1985, ApJ, 295, 143

Paczyński, B. 1971, ARA\&A, 9, 183

Remillard, R. A., \& McClintock, J. E. 2006, ARA\&A, 44, 49

Scargle, J. D. 1981, ApJS, 45, 1

Shakura, N. I., \& Sunyaev, R. A. 1973, A\&A, 24, 337

Shappee, B. J., Prieto, J. L., Grupe, D., et al. 2014, ApJ, 788, 48

Voges, W., Aschenbach, B., Boller, T., et al. 2000, IAUC, 7432, 3

Ziaali, E., Bedding, T. R., Murphy, S. J., et al. 2019, MNRAS, 486, 4348

Zong, W., Fu, J.-N., De Cat, P., et al. 2018, ApJS, 238, 30 